\title{
Spatial Graphs for Intra-cranial Vascular Network Characterization, Generation, and Discrimination
}

\author{
Stephen R. Aylward ${ }^{1}$, Julien Jomier ${ }^{1}$, Christelle Vivert ${ }^{1}$, \\ Vincent LeDigarcher ${ }^{1}$, and Elizabeth Bullitt ${ }^{2}$ \\ 1 CADDLab, Department of Radiology \\ aylward@unc.edu \\ 2 Department of Surgery, University of North Carolina, USA
}

\begin{abstract}
Graph methods that summarize vasculature by its branching topology are not sufficient for the statistical characterization of a population of intra-cranial vascular networks. Intra-cranial vascular networks are typified by topological variations and long, wandering paths between branch points.

We present a graph-based representation, called spatial graphs, that captures both the branching patterns and the spatial locations of vascular networks. Furthermore, we present companion methods that allow spatial graphs to (1) statistically characterize populations of vascular networks, (2) generate the central vascular network of a population of vascular networks, and (3) distinguish between populations of vascular networks. We evaluate spatial graphs by using them to distinguish the gender and handedness of individuals based on their intra-cranial vascular networks.
\end{abstract}

\section{Introduction}

Intra-cranial vasculature varies across individuals to such an extent that only a few of the largest intra-cranial vessels are sufficiently consistent to depicted and labeled in anatomical atlases 13 . Variations in the location and connectivity of even those named vessels are common in a healthy population [2]. Furthermore, between branchpoints, intra-cranial vessels will often follow long and wandering walks. These walks must be preserved to truly capture the form and function (filling regions, etc.) of intra-cranial vasculature. Our interest in characterizing intra-cranial vasculature arises from the mounting evidence that a genetic relationship exists between mental disorders and vascular network formation 10, 15.

We have developed a novel, graph-theoretic method for representing and analyzing the intra-cranial vascular networks of individuals and populations. Our "spatial graphs" capture the anatomical locations, branching patterns, and tortuous paths of intra-cranial vascular networks. Spatial graph formation begins with a centroid voronoi tessellation (CVT) 11] of intra-cranial space. Those CVT regions are potential nodes of a spatial graph. A vascular network, overlaid on a voronoi tessellation of space, defines a spatial graph by recording the branching sequence of CVT nodes visited by the network. These branching sequences can 
be equivalently recorded as weighted, asymmetric adjacency matrices. Adjacency matrix and node-specific statistics, such as the centrality [3] and branch probability at each node, can be collected for a population of vascular networks. We have defined a method whereby those statistics can be used to probabilistically generate vascular networks that are central to a population. We have also developed a method with which those statistics can be used to compute membership scores for an individual's vascular network; an individual's membership scores from different population-specific graphs and be used to classify that individual.

Forming graph representations of vascular networks is not a new concept. In 1993 Dr. Gerig presented [9] an intra-cranial vessel segmentation technique and illustrated the reduction of those segmentations to graphs that represent their topology. Such branch-based graphs are the basis of much of the vessel-based liver lobe and heart vessel segmentation work being conducted in a variety of labs 8 [14. In computer-vision, graph theory has been applied to the characterization of the medial structure of objects [12. To the best of our knowledge, a method has not been previously demonstrated that applies graph theory to the characterization, generation, and discrimination of populations of intra-cranial vascular networks.

In the next section we present our methods. The subsequent section describes three evaluations of our methods: (1) visually assessing the central vascular network generated from the spatial graph statistics from right-handed males, (2) using male and female-specific spatial graphs of intra-cranial vasculature to determine the gender of testing individuals, and (3) using a right-handed-specific population graph to determine the handedness of testing individuals.

\section{Methods}

This section is divided into three subsections: forming spatial graphs, generating vascular networks that are central to population of vascular networks, and computing graph membership measures. While these method descriptions focus on intra-cranial vasculature, these methods are applicable to any branching, directed, cyclic, space-occupying structure.

\subsection{Forming Spatial Graphs}

Spatial graph formation involves vessel extraction, inter-subject registration, space partitioning, and recording graph statistics. These steps are detailed next.

1) Extract the vascular networks from the individuals' MRA data. These encodings must capture the location of vessels, their directions of blood flow, and their branchpoints to accurately encode a spatial graph as a directed graph. We have developed a vessel network that has been shown to produce accurate localizations [1] and accurate flow direction and branch point information [4. Our method works for contrast CT and time-of-flight MR angiograms (MRA).

2) Align the individuals' vascular networks to a common coordinate frame. We achieve this by (a) rigidly registering, using mutual-information, the individuals' MRA to their T1 images; (b) performing an affine, mutual information 
registration between the T1 images and the BrainWeb T1 atlas [7] (c) composing the corresponding MRA-to-T1 and T1-to-atlas transforms; and (d) applying the composed transform to the extracted vessels. [6]

3) Partition intra-cranial space. The goal of this step is to define a partitioning that captures vascular locations, branches, and paths in intra-cranial space. This information is best captured when each partition has equal likelihood of containing a vessel; otherwise under-utilized partitions would offer less discriminatory information. We therefore partition space using expected vascular density as follows.

Expected vascular density is computed using the vascular distance maps for a population [6]. Specifically, for each vascular network, the squared distance to the nearest vessel is computed throughout intra-cranial space. Those distance maps are then inverted so that points nearer the vessels have larger values; thus, intensity is related to likelihood of finding a vessel at that point. A mean distance map is computed as a voxel-by-voxel average of those maps.

An equi-probable partitioning of expected vascular density is computed using centroid voronoi tessellation (CVT) [11. For this paper, $1000 \mathrm{CVT}$ regions are resolved using a probabilistic version of Lloyd's method [11. This algorithm is computed for 1000 iterations using 100,000 new, randomly generated samples per iteration. Random samples are generated from the mean density map using the rejection method [11], so, the likelihood of selecting a voxel as a sample is proportional to the image intensity at that voxel. Each iteration, the mass centroids of the voronoi regions are updated using a new set of samples. Results are illustrated in Fig. 1.

4) Recording graph statistics. The adjacency matrix and node-specific statistics summarize how vascular networks map onto the CVT regions.

For adjacency matrix computation, as each vessel's directed traversal crosses from CVT region $i$ to the next region $j$, the corresponding position $A_{i j}$ in the

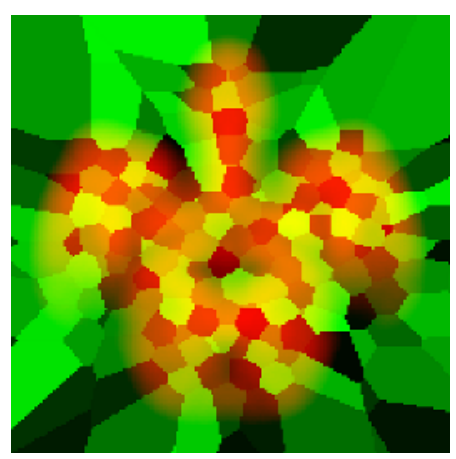

Fig. 1. An axial slice (at the level of the Circle of Willis) through the mean vessel distance map and CVT partitioning formed from 18 individuals. The fuzzy (red) object in the background is the expected vascular density. The crisp (green) overlay uses random intensities to depict the computed CVT regions. 
population's adjacency matrix is increased by $1 /$ (total number of vessels in that individual's network). This weighting compensates for level-of-detail variations in the vascular networks.

Node statistics summarize local and connectivity information. At each node we record (a) the number of times it contains a branch point, (b) the mean radius of the vessel points that are contained within it, and (c) the number of times a root artery, e.g., a carotid or basilar artery, originates from within it. Additionally, in this paper, we compute node centrality as a local summary of connectivity. Our node centrality computation is an adaptation of a method developed for determining the "popular people" in a social network, i.e., the most "central person" in directed communications [3. To compute such centrality, a value $e_{i}$ represents the externally defined status of person $i$ in the network. A parameter $\alpha$ is set to weight the influence of that external status on internal exchanges. The centrality $c_{i}$ of a node/person is then computed as

$$
c=\left(I-\alpha A^{t}\right)^{-1} e
$$

where $A$ is the asymmetric adjacency matrix and $I$ is the identity matrix. We applied this measure to our vascular networks by giving every CVT node equal external status, i.e., $e_{i}=1$ for all $i$, and setting $\alpha=0.01$ to reduce the influence of external status. In the future, it may be useful to relate external status to the volume of a vessel's fill region or its radius. We also removed the transpose of $A$ so that the directed links in our networks are inverted; all vessels are made to flow to their roots, e.g., the carotids. After applying Equ. 1 the node that most often contains the root of the largest tree receives the largest centrality value. The next distal node is the adjacent node with the highest centrality value, and so forth. Using adjacency and node statistics, vascular graphs can be generated and classified, as described next.

\subsection{Generating Central Vascular Networks}

Generating the most central vascular network from a population's spatial graph involves a greedy traversal of the population's adjacency matrix and the consideration of the branch probabilities at its nodes. Since each node correspond to a volume in space, a branching sequence of nodes defines a branching network passing through those volumes in space. Our generation process involves two user specified threshold values. The first threshold $\theta_{A}$ indicates the minimum level of adjacency required for a vessel traversal to continue. The second threshold $\theta_{b}$ indicates the minimum branch-support required for a branch point to be taken. Branch-support from a node $i$ to node $j$ is defined by the product of the probability of branching at node $i$ and the adjacency weight from $i$ to $j$.

Generation begins at the most likely root node, as reported by the recorded node statistics. The largest weighted adjacency from that node is then chosen as the next transition in the generated graph. Adjacent nodes, not selected for continued generation, are searched for branch-support values above $\theta_{b}$. If a branch node is found, it is queued for generation after the current vessel's generation 
terminates. A vessel's generation terminates when it encounters a node from which none of the adjacent nodes have an adjacency value above $\theta_{A}$. Future research should consider statistics regarding radius, mean curvature, etc. when generating vessels.

\subsection{Graph Membership Measures}

Membership scores for a new vascular network, represented by the spatial graph $g$, are conditional on a population's spatial graph $G$. We evaluate two membership scores in this paper: $\Sigma_{A}(g \mid G)$ is the sum of the adjacency weights in $G$ for all transitions that also exist in $g$, and $\Sigma_{c}(g \mid G)$ is the sum of the centrality values for the CVT nodes in $G$ that are also touched by $g$. Both of these values should be maximized when a vascular network is compared with a graph that represents the population to which that vascular networks belongs. The next section describes experiments which test this theory.

\section{Results}

Three experiments were conducted to evaluate spatial graphs. Each of these is an unsolved problem in medical image analysis.

For these experiments, MRA and T1-weighted MR images were collected on a Siemens Allegra head-only $3 \mathrm{~T}$ MR system. MRA data was acquired using a time-of-flight sequence at $0.5 \times 0.5 \times 0.8 \mathrm{~mm}^{3}$ voxel spacing. T1-weighted MR data was acquired at $1 \times 1 \times 1 \mathrm{~mm}^{3}$.

The images from an individual were either used for training or testing, but never for both. Individuals were labaled by gender and handedness.

All of the experiments began with a common CVT partitioning of intracranial space. Specifically, vascular networks from 9 training-data, right-handed males (ages 22-55, mean age 34.4) and 9 training-data, right-handed females (ages 20-54, mean age 35.3) were combined to produce a common mean density map. The CVT of that map was computed once. Adjacency matrices, node centrality, and other measures were computed using the designated individuals' vascular networks combined with this common CVT partitioning. Using a common CVT partitioning may have reduced sensitivity and specificity, but it allowed node statistics to be compared across the populations' spatial graphs. This CVT partitioning is depicted in Fig. 1

\subsection{Experiment 1: Vascular Graph Generation}

The first experiment demonstrates the generation of the central vascular network for right-handed males. Using the 9 training-data, right-handed males, a righthanded, male population spatial graph was computed. The vascular network generation method described above was then applied. For visualization, the midpoints on the lines between the centroids of each generated sequence of nodes were used as the control points for a b-spline curve through space. A constant radius of $0.5 \mathrm{~mm}$ was used for visualizing the vessels as tubes. 

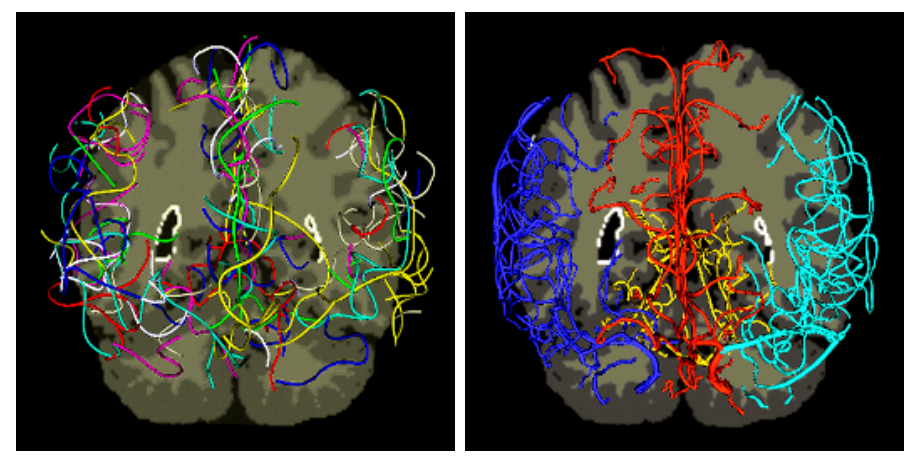

Fig. 2. Left: A vascular network generated from the right-handed male graph. Right: A vascular network from one of the training right-handed males. Carotids, circle of willis, vascular groups, other vascular patters are evident in the generated network.

The generated central vascular network is visualized in Fig. 2. Subjectively, there are striking similarities between the generated and actual intra-cranial vascular networks. The generation method's threshold values can be manipulated to produce various levels of branching detail. The image shown used a low branching threshold, $\theta_{b}=0.001$, so as to simulate extensive branching detail. Major vascular groups, the carotids, circle of willis, basilar artery, communicating arteries, and other major vessels are evident. Using b-splines to visualize the node adjacency sequence may have exaggerated intra-node vessel curvature.

\subsection{Experiment 2: Distinguishing Gender}

The second experiment involved distinguishing males from females based on intra-cranial vasculature. Two population-specific spatial graphs were computed: one using 9 training-data, right-handed males and the other using 9 trainingdata, right-handed females. For each population-specific graph, the membership

Table 1. (Upper Table) Mean difference for each membership measure for testing individuals given gender-specific population graphs. (Lower Table) Mean differences for each membership measure for testing individuals given right-handed and mirroredright-handed population graphs. Items in bold highlight population-specific graphs that correctly classified every individual in the corresponding testing data.

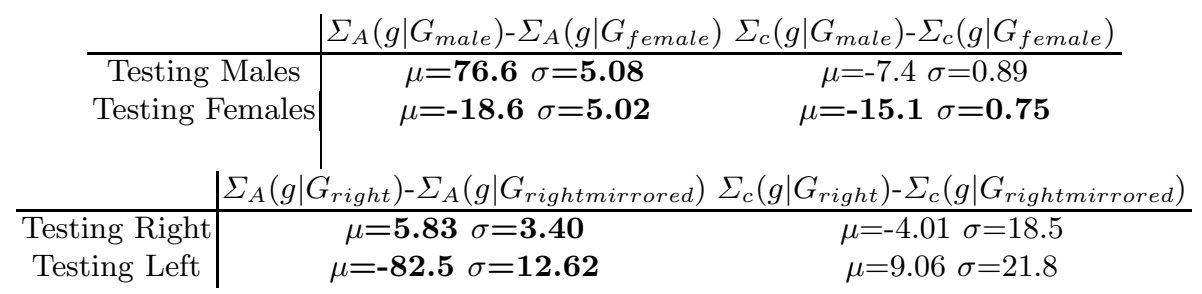


measures $\Sigma_{A}(g \mid G)$ and $\Sigma_{c}(g \mid G)$ were computed for 3 testing-data, right-handed males (ages 23, 26, 57) and 3 testing-data, right-handed females (ages 33, 43, and 57).

Gender classification results are shown in Tab. 11 The $\Sigma_{A}$ measure produced correct classifications for every testing-data male and every testing-data female. Results for $\Sigma_{c}$ contained $50 \%$ classification error: mislabeling every testing-data male as female. The success of $\Sigma_{A}$ and failure of $\Sigma_{c}$ implies that gender differences are not based on the location or number of vessels, but it is the directed vascular paths throught space that distinguish males from females. Further research is focusing on visualizing these differences.

\subsection{Experiment 3: Determining Handedness}

Our third experiment involved analyzing brain lateralization to determine the self-reported handedness of individuals. To study brain lateralization, instead of creating a population-specific spatial graph for left-handed males, we mirrored the right-handed graph along its x-axis. This mirroring approximates brain lateralization since the BrainWeb atlas' mid-sagittal plane is at the center of and normal to the $\mathrm{x}$-axis. Using these right-handed male and mirrored-righthanded-male population-specific spatial graphs, membership measures $\Sigma_{A}(g \mid G)$ and $\Sigma_{c}(g \mid G)$ were computed for the 3 testing-data right-handed males and for 3 testing-data left-handed males (ages 19, 26, and 29).

Results from handedness classification using intra-cranial vasculature are shown in Tab. 1. Again the adjacency membership measure $\Sigma_{A}(g \mid G)$ lead to correct labels for all testing-data individuals. The centrality measure $\Sigma_{c}(g \mid G)$ had a $66 \%$ classification error; mislabeling two right-handed individuals as lefthanded and two left-handed individuals as right-handed. Again, the success of $\Sigma_{A}(g \mid G)$ and the failure of $\Sigma_{c}(g \mid G)$ suggests that the direct paths of intra-cranial vasculature is key to distinguishing these populations.

\section{Discussion}

We have developed spatial graphs as a technique which is well suited for characterizing intra-cranial vasculature. Initial results indicate that spatial graphs can be used to classify the gender and handedness of individuals. These successes suggests that spatial graphs can be used to study the genetic relationship that may exists between mental disorders and vascular network formation [10] 15].

One particularly interesting finding is that when various membership measures are compared, classification experiments suggest that it is the paths taken by the vessels in a network (their adjacency matrices), not their centrality, that distinguishes genetic vascular variations.

While these results are encouraging, we are very cautious about drawing any conclusions beyond the limited data involved. It is critical that these methods be applied to larger datasets before suggesting that any general "truths" have been revealed. Future work will also pursue more advanced statistical analysis techniques. 


\section{References}

1. Aylward, S and Bullitt, E, Initialization, Noise, Singularities and Scale in Height Ridge Traversal for Tubular Object Centerline Extraction, IEEE TMI 21(2):61-75, 2002

2. Baskaya, MF; et al. Surgical and Angiographic Anatomy of the Posterior Communicating and Anterior Communicating Arteries, Neuro-anatomy 3:38-42, 2004

3. Bonacich, P and Lloyd, P, Eigenvector-Like Measures of Centrality for Asymmetric Relations, Social Networks 23:191-201, 2001

4. Bullitt, E; Aylward, S; Liu, A; Stone, J; Mukherji, S; Coffey, C; Gerig, G; Pizer, SM, 3D Graph Description of the Intracerebral Vasculature from Segmented MRA and Tests of Accuracy by Comparison with X-Ray Angiograms, IPMI:308-321, 1999

5. Bullitt, E; Aylward, S; Smith, K; Mukherji, S; Jiroutek, M; and Muller, K, Symbolic Description of Intracerebral Vessels Segmented From MRA and Evaluation by Comparison with X-Ray Angiograms, MedIA 5:157-169, 2001

6. Chillet, D; Jomier, J; Cool, D; Aylward, S, Vascular Atlas Formation Using a Vessel-to-Image Affine Registration Method, MICCAI :335-342, 2003

7. Cocosco, CA; Kollokian, V; Kwan, RK-S; Evans, AC, BrainWeb: Online Interface to a 3D MRI Simulated Brain Database NeuroImage 5(4), 1997

8. Ezquerra, N; Capell, S; Klein, L; Duijves, P, Model-Guided Labeling of Coronoary Structure, IEEE TMI 17(3):429-441, 1989

9. Gerig, G; Koller, T; Székely, G; rechbühler, C; übler, O, Symbolic Description of 3-D Structures Applied to Cerebral Vessel Tree Obtained From MR Angiography Volume Data, IPMI :94-111, 1993

10. Hesser, BA; et. al, Down Syndrome Critical Region Protein 1 (DSCR1), a Vovel VEGF Target Gene That Regulates Expression of Inflammatory Markers on Activated Endothelial Cells, Blood 104(1):149-158, 2004

11. Ju, L; Du, Q; Gunzburger, M, Porbabilistic Methods for Centroidal Voronoi Tessellations and Their Parallel implementations, Parallel Computing 28:1477-1500 2002

12. Macrini, D,; Shokoufandeh, A; Dickinson SJ; Siddiqi, K; Zucker; SW, View-Based 3-D Object Recognition Using Shock Graphs, ICPR:24-32, 2002

13. Netter, F, Atlas of Human Anatomy, Novartis, East Hanover, new Jersey, pages 525,1989

14. Selle, D; Preim, B; Schenk, A; Peitgen, H.-O., Analysis of Vasculature for Liver Surgical Planning IEEE TMI 21(11):1344-1357, 2002

15. Stalmans, I; et al., VEGF: A Modifier of the del22q11 (DiGeorge) Syndrome? Nature Medicine 9:173-182, 2003 